\title{
س
}

$>\mathrm{DE}$

$\approx$ PÉDAGOGIE

\author{
Recherches en éducation
}

172 | juillet-septembre 2010

La pédagogie universitaire : un courant en plein développement

\section{BAUTIER Élisabeth \& RAYOU Patrick. Les inégalités d'apprentissage. Programmes, pratiques et malentendus scolaires}

Paris : PUF, 2009, 184 p.

Mathias Millet

\section{(2) OpenEdition \\ Journals}

Édition électronique

URL : http://journals.openedition.org/rfp/2311

DOI : 10.4000/rfp.2311

ISSN : 2105-2913

Éditeur

ENS Éditions

Édition imprimée

Date de publication : 15 juillet 2010

Pagination : 133-134

ISBN : 978-2-7342-1188-4

ISSN : 0556-7807

\section{Référence électronique}

Mathias Millet, «BAUtIER Élisabeth \& Rayou Patrick. Les inégalités d'apprentissage. Programmes, pratiques et malentendus scolaires », Revue française de pédagogie [En ligne], 172 I juillet-septembre 2010, mis en ligne le 24 janvier 2011, consulté le 25 septembre 2020. URL : http://journals.openedition.org/rfp/2311 ; DOI : https://doi.org/10.4000/rfp.2311

Ce document a été généré automatiquement le 25 septembre 2020.

(c) tous droits réservés 


\section{BAUTIER Élisabeth \& RAYOU Patrick. Les inégalités d'apprentissage. Programmes, pratiques et malentendus scolaires}

Paris : PUF, 2009, $184 \mathrm{p}$.

Mathias Millet

\section{RÉFÉRENCE}

BAUTIER Élisabeth \& RAYOU Patrick. Les inégalités d'apprentissage. Programmes, pratiques et malentendus scolaires. Paris : PUF, 2009, $184 \mathrm{p}$.

1 Le livre d'Élisabeth Bautier et de Patrick Rayou constitue la synthèse d'un ensemble de travaux (les leurs et ceux qui leur font écho) sur les "phénomènes d'apprentissage différenciés " dont ils montrent la cohérence et la pertinence pour l'analyse des "processus sociaux par lesquels les élèves apprennent ou n'apprennent pas selon les normes scolaires ». Il ne s'agit donc pas de la présentation des résultats d'une enquête empirique particulière. Plusieurs choix descriptifs structurent le propos de l'ouvrage. Le premier est de couvrir tout le spectre du système éducatif. Loin de se focaliser sur la seule école primaire comme le font nombre d'études centrées sur la question des apprentissages scolaires, l'ouvrage cherche à dégager des processus transversaux dans les scolarités "défaillantes", de la maternelle à l'université. Le second réside dans l'attention particulière accordée aux dimensions sociolangagières (discours pédagogique, usage de la langue dans les classes, littératie étendue, etc.) comme analyseur du rapport d'apprentissage. Le caractère exemplaire de ces dimensions dans l'appréhension des inégalités scolaires, et les orientations empiriques des auteurs, en fait un vecteur de démonstration empirique récurrent, présent tout au long de l'ouvrage. Le troisième développe une perspective à la fois historique, sociologique et 
psychologique qui, dans l'analyse de ce qui fait les inégalités et difficultés d'apprentissage, articule l'effet des transformations générales de l'école, leurs conséquences sur la relation pédagogique et les pratiques de classes et la question des épreuves « subjectives » que les situations scolaires constituent pour les élèves.

2 C'est la notion de malentendu, central à tout l'ouvrage, qui constitue le fil directeur de l'argumentation. Son intérêt heuristique est d'éviter de réduire les difficultés scolaires à des incapacités cognitives ou à un désintérêt scolaire des élèves. Elle conduit à penser au contraire les différences d'apprentissage comme des « constructions conjointes » de l'enseignant et de l'élève, en confrontant les situations mises en place par les enseignants pour apprendre et les interprétations ou détournements qu'en font les élèves. La notion offre un renversement de perspective intéressant : ce n'est plus ainsi "parce que les élèves n'apprennent pas, ne travaillent pas qu'ils ont des difficultés, c'est parce qu'il y a ces malentendus que les apprentissages ne peuvent s'effectuer [...]. » Tout l'ouvrage consiste à restituer les conditions de ces malentendus qui font les difficultés scolaires des élèves, et à éclairer leurs incarnations concrètes dans les classes autour de causes à la fois sociales, historiques et pédagogiques.

3 Élisabeth Bautier et Patrick Rayou défendent l'idée selon laquelle la scolarisation de masse, en changeant à la fois les publics comme les savoirs scolaires, est indissociable d'un ensemble de brouillages de la forme scolaire. C'est ainsi, par exemple, qu'en réaffirmant les missions intégratives de l'école, la massification s'est parfois traduite en une série de renoncements scolaires, c'est-à-dire par une adaptation à la baisse des programmes dispensés aux élèves des quartiers populaires ou par l'adoption de méthodes d'enseignement basées sur l'image, le jeu ou la théâtralisation davantage que sur la conceptualisation. De même la levée progressive, dans l'école, des obstacles sociaux qui empêchaient certains élèves d'en franchir les étapes a-t-elle eu pour conséquence de retourner contre la personne des élèves, dans une logique responsabilisante et individualisante, le résultat de leurs échecs. Au-delà, ce sont les transformations des matrices scolaires que l'ouvrage pointe comme étant au principe d'une forme scolaire devenue moins lisible. La promotion d'une forme scolaire moins fermée dans l'espace et le temps spécifiques de sa relation et de ses savoirs a eu pour effet de rendre celle-ci plus perméable "à des savoirs, des comportements, des influences locales, des objets qu'elle avait contenus à sa frontière, voire annexés. » Or, en promouvant la scolarisation des objets de l'expérience commune, source supposée de motivation et de facilitation scolaires, l'école a aussi rendu plus indistincte la frontière entre l'expérience commune et l'expérience scolaire et contribué à altérer l'identification des tâches scolaires.

4 À cet égard, les auteurs de cet ouvrage montrent que l'ouverture scolaire est indissociable de "bougés curriculaires » et pédagogiques dont il faut prendre la pleine mesure pour saisir les mécanismes de production des malentendus sociocognitifs et des difficultés d'apprentissage. Car l'ensemble de ces évolutions tant curriculaires que pédagogiques vient contredire la figure de l'élève en formation, autonome, figure effectivement visée par l'école, fondée sur la maîtrise d'une « littératie étendue » (c'està-dire d'une langue et d'une raison ancrées dans les habitudes de l'écrit généralisé qui caractérise nos sociétés contemporaines). La préconisation du débat et les échanges langagiers qui trament désormais le quotidien des classes, sur lesquels les auteurs s'arrêtent longuement, en témoignent. Sous couvert d'un oral de la "quotidienneté » ordinaire par l'intermédiaire duquel l'école tente d'amener les élèves à parler coûte que 
coûte, c'est pourtant toujours la maîtrise d'une langue secondarisée ou scripturale scolaire qui est attendue. Or ces situations de langage accréditent l'idée, chez les élèves, que prévalent «les caractéristiques linguistiques et langagières d'une communication dans l'entre-soi, où chacun parle au nom de ce qu'il est autant que de ce qu'il sait, où l'enseignant dit "nous" en s'intégrant ainsi au groupe, dans un statut et un rôle indistincts de ceux des élèves, l'âge seul pouvant justifier une expérience et des connaissances plus larges." De mêmes analyses sont encore proposées en ce qui concerne les situations scolaires de travail à faible cadrage, faiblement contrôlées, où l'apprentissage par la pédagogie du "faire " conduit les élèves à percevoir l'école comme une succession de choses à faire, sans lien les unes avec les autres, dans le cadre de laquelle "faire et voir se sont peu à peu substitués à apprendre, enseigner, expliquer, travailler, dans la langue même censée décrire et dire les activités scolaires. »

5 L'ensemble de ces analyses, et bien d'autres encore, permettent de mettre en relation "les inégalités potentielles d'apprentissage avec des évolutions curriculaires, des changements qui concernent les principes de clôture de l'école à l'égard de l'extérieur, les modes de faire la classe qui valorisent les échanges langagiers et certaines formes individualisées du travail de l'élève. » Au final, le livre constitue un bel exemple de sociologie cognitive appliquée aux questions scolaires. Contrairement aux analyses qui négligent la question de la transmission et de l'appropriation des savoirs scolaires, celles-ci se centrent sur la nature et les modalités de ce qui y est enseigné et les relations d'apprentissage qui font la matière principale de l'école et s'avèrent indispensables à la compréhension des mécanismes différenciés d'appropriation des savoirs et des situations scolaires. Pour autant, l'ouvrage n'évacue pas complètement une analyse plus classique en termes d'inégalités socioculturelles. Est par exemple rappelée, en référence notamment aux travaux sur la forme scolaire, l'existence de tensions socialisatrices entre le mode scolaire de socialisation et les logiques socialisatrices dans les familles populaires. Poursuivant le débat entre Samuel Johsua et Bernard Lahire dans la revue Éducation et sociétés ( ${ }^{\circ} 4$ de 1999), les auteurs s'efforcent ainsi de lier approche didactique et approche sociologique. D'un côté, il s'agit de saisir les gestes d'apprentissage sans raisonner sur un élève moyen; de l'autre, de prêter attention aux mécanismes sociaux de différenciation scolaire sans omettre la question, centrale, des modalités d'apprentissage.

\section{AUTEURS}

\section{MATHIAS MILLET}

GRESCO, université de Poitiers 